\title{
Lenalidomide improvement of cisplatin antitumor efficacy on triple-negative breast cancer cells in vitro
}

\author{
LIN-LIN YIN ${ }^{1,2}$, XIN-MIAN WEN ${ }^{3}$, QING-HUA LAI ${ }^{4},{\text { JING } L^{2}}^{2}$ and XIU-WEN WANG ${ }^{1}$ \\ ${ }^{1}$ Department of Oncology, Qilu Hospital of Shandong University, Jinan, Shandong 250012; \\ Departments of ${ }^{2}$ Hematology and Oncology, and ${ }^{3}$ Clinical Laboratory, The Fourth Hospital of Jinan, Jinan, \\ Shandong 250031; ${ }^{4}$ Department of Oncology, The Central Hospital of Jinan, Jinan, Shandong 250013, P.R. China
}

Received August 11,2017; Accepted February 9, 2018

DOI: $10.3892 / \mathrm{ol} .2018 .8120$

\begin{abstract}
Lenalidomide is an immunomodulatory drug and possesses anti-angiogenic and immunomodulatory activities against multiple myeloma. The present study assessed the in vitro effect of lenalidomide combined with cisplatin on MDA-MB-231, a triple-negative breast cancer (TNBC) cell line and explored the underlying molecular mechanism of this combination. Cell viability, apoptosis and the protein expression of phosphorylated (p) and total extracellular signal-regulated kinase (ERK), B-cell lymphoma-2 (Bcl-2), caspase-3, cleaved poly-adenosine diphosphate-ribose polymerase (cPARP), vascular endothelial growth factor (VEGF) and basic fibroblast growth factor (bFGF) were measured in MDA-MB-231 cells treated with different concentrations of lenalidomide, cisplatin and their combination using different biochemical assays. Lenalidomide demonstrated no significant effect on the cell viability of MDA-MB-231 cells, even at high concentrations, whereas lenalidomide in combination with cisplatin, significantly reduced cisplatin $\mathrm{IC}_{50}$ from 7.8 to $3.0 \mu \mathrm{M}$ in MDA-MB-231 cells. In addition, lenalidomide and cisplatin in combination significantly induced cell apoptosis by 1.6 and 1.38 -fold, respectively compared with lenalidomide and cisplatin alone $(\mathrm{P}<0.05)$. The expression levels of VEGF, bFGF and $\mathrm{Bcl}-2$ proteins were significantly reduced $(\mathrm{P}<0.01)$, whereas caspase- 3 and cleaved PARP expression were significantly increased in MDA-MB-231 cells treated with the combination compared to those treated with single agents $(\mathrm{P}<0.01)$. Lenalidomide treatment alone significantly reduced the p-ERK level compared with the control $(\mathrm{P}<0.05)$ and
\end{abstract}

Correspondence to: Dr Jing Li, Department of Hematology and Oncology, The Fourth Hospital of Jinan, 50 Shifan Road, Jinan, Shandong 250031, P.R. China

E-mail: leejing1967@163.com

Dr Xiu-Wen Wang, Department of Oncology, Qilu Hospital of Shandong University, 44 Wenhua Xi Road, Jinan, Shandong 250012, P.R. China

E-mail: enthusim@hotmail.com

Key words: lenalidomide, cisplatin, triple-negative breast cancer, apoptosis cisplatin treatment alone significantly increased it $(\mathrm{P}<0.01)$, however treatment with them in combination significantly reduced the p-ERK level in MDA-MB-231 cells compared with cisplatin treatment alone $(\mathrm{P}<0.05)$. In conclusion, the present study provides the basis for using lenalidomide in combination with cisplatin in TNBC therapy.

\section{Introduction}

Breast cancer is a significant worldwide health problem in women and accounts for $12 \%$ of all cancers with 1.7 million newly diagnosed cases and $6 \%$ of all cancer deaths globally in 2012 (https://www.cdc.gov/cancer/international/statistics-htm. Retrieved on March 30, 2017). Fortunately, decades-long advancement in breast cancer treatment and prevention lead to dramatic improvement of breast cancer patient's survival. According to St Gallen Conference 2013, breast cancer can be molecularly classified into different subtypes, including luminal A (ER and PR-positive, low rate of Ki-67 and HER2-negative) and luminal B, luminal B1, HER2-negative (ER-positive, PR $<10 \%$ or negative, high rate of Ki-67), luminal B2 HER2-positive (ER-positive and PR-negative), HER2-positive non-luminal (ER and PR-negative) and basal-like (ER, PR and HER2-negative) (1). The receptors-positive breast cancer is usually cured with anti-receptor therapy (e.g., tamoxifen treatment for ER+ breast cancer, while Trastuzumab for HER2 ${ }^{+}$breast cancer (2-4). The basal-like subtype, also known as triple negative breast cancer (TNBC), is commonly negative for ER, PR and HER2. However, TNBC, represents $15 \%$ of all breast cancer, often occurs in young women and $80-90 \%$ of TNBC is an aggressive invasive ductal carcinoma (5), while $15-30 \%$ of TNBC patients will eventually develop tumor brain metastasis (6). To date, there is no effective therapy for TNBC and poor survival is common in TNBC compared to other breast cancer subtypes. Thus, effective therapy that prolongs or improves survival of TNBC patients is needed.

Lenalidomide is a derivative of thalidomide, which is an immunomodulatory drug. Lenalidomide has been reported to possesses anti-angiogenic and immunomodulatory effects (7-10) and was approved by the US FDA as the first-line therapy in multiple myeloma (11). Recent clinical trials have demonstrated the antitumor effect of lenalidomide 
in different solid tumors, such as hepatocellular cancer (12) and colorectal cancer (13). In addition to the anti-angiogenic and immunomodulatory properties, lenalidomide has been shown to have cytotoxic effect in tumor cells (13). In clinical trials, lenalidomide was either used as a single agent or in combination with other chemotherapeutic drugs in many solid tumors; for example, Said et al showed that lenalidomide in combination with 5-fluorouracil, leucovorin, and oxaliplatin was able to control advanced colorectal cancer (13), while Safran et al assessed the activity and efficacy of lenalidomide on advanced hepatocellular cancer that was previously treated with sorafenib (14), where six out of 40 patients (15\%) achieved a partial response, including two patients $(5 \%)$ had stable disease for more than 32 months (14). These data indicate that lenalidomide could be more effective in combination with chemotherapeutic drugs, such as gemcitabine or docetaxel $(12,15)$. Furthermore, Brosseau et al (16) demonstrated that lenalidomide was able to inhibit proliferation of MDA-MB-231 cells through the restoration of vitamin D sensitive phenotype. Wu et al (12) also showed that combination of lenalidomide with gemcitabine improved survival of pancreatic cancer patients. Thus, in the present study, we will investigate the therapeutic effect of lenalidomide on TNBC cells in combination with cisplatin, a frequently used chemotherapeutic drug for TNBC. The concentration of drug required to reduce cell viability by $50 \%\left(\mathrm{IC}_{50}\right)$ was calculated using Compusyn software (ComboSyn, Inc., Paramus, NJ, USA) (17). We expected that lenalidomide could reduce of cisplatin, reducing cisplatin $\mathrm{IC}_{50}$ value, inducing tumor cells to undergo apoptosis, and inhibiting angiogenesis in triple-negative breast cancer MDA-MB-231 cells in vitro.

\section{Materials and methods}

Reagents and cell culture. Lenalidomide was purchased from the AMQUAR Corporation (cat. no. EY0006; Denver, CO, USA), while cisplatin was obtained from MCE Corporation (cat. no. HY-17394; Dublin, CA, USA). Lenalidomide was dissolved in dimethyl sulfoxide (DMSO) at $10 \mathrm{mM}$ stock solution, stored at $-20^{\circ} \mathrm{C}$, and used within a week. Cisplatin was dissolved in distilled water at $10 \mathrm{mM}$ stock solution and stored at $4^{\circ} \mathrm{C}$.

A human triple-negative breast cancer line MDA-MB-231 was obtained from the Chinese Academy of Sciences (Shanghai, China) and maintained in Dulbecco's modified Eagle's medium (Thermo-Fisher, Waltham, MA, USA) supplemented with $10 \%$ fetal bovine serum and $1 \%$ penicillin-streptomycin (both from Gibco, Gaithersburg, MD, USA) in a humidified incubator with $5 \% \mathrm{CO}_{2}$ at $37^{\circ} \mathrm{C}$.

Cell viability MTT assay. Cell viability was measured using the 3-(4,5-dimethylthiazol-2-yl)-2,5-diphenyltetrazolium bromide (MTT; Sigma-Aldrich, Merck KGaA, Darmstadt, Germany) assay. In brief, MDA-MB-231 cells were seeded into 96-well plates at a density of $5 \times 10^{3}$ per well and cultured overnight. On the next day, cells were treated with various concentrations of cisplatin $(0.12-30 \mu \mathrm{M})$, lenalidomide $(1.25-320 \mu \mathrm{M})$ or their combination in $100 \mu \mathrm{l}$ volumes of growth medium for $72 \mathrm{~h}$ at $37^{\circ} \mathrm{C}$. After that, $10 \mu \mathrm{l}$ of MTT was added to each well and further incubated for $4 \mathrm{~h}$. Further, the medium was replaced with $150 \mu 1$ of DMSO in each well and thoroughly mixed and then the optical absorbance value was measured at $570 \mathrm{~nm}$ using a plate reader (Eppendorf, Hamburg, Germany). The control cells were treated with $100 \mu 1$ of phosphate-buffered saline (PBS) or $1 \%$ of DMSO. The $\mathrm{IC}_{50}$ values of cisplatin, lenalidomide, and their combination were calculated using the Compusyn software version 1.0 (ComboSyn, Inc., Paramus, NJ, USA) as described previously (17).

Flow cytometric apoptosis assay. To assess cell apoptosis, MDA-MB-231 cells were seeded into 6-well plates at a density of $1 \times 10^{5}$ per well. After $24 \mathrm{~h}$ culture, cells were treated with cisplatin $(3 \mu \mathrm{M})$, lenalidomide $(1 \mu \mathrm{M})$, or their combination for $72 \mathrm{~h}$. Cells were then collected in ice-cold PBS, and apoptotic cells were detected using FITC Annexin V-FITC/PI Apoptosis Detection kit (cat. no. 4A; Biotech Co., Ltd., Beijing, China) according to the manufacturer's protocol. The stained cells were then measured with a flow cytometer (BD Biosciences, Franklin Lakes, NJ, USA).

Protein extraction and western blot analysis. MDA-MB-231 cells were seeded into 6-well plates and they were let grow up to $80 \%$ confluency. The medium was replaced with $3 \mu \mathrm{M}$ cisplatin, $1 \mu \mathrm{M}$ lenalidomide or their combination for $72 \mathrm{~h}$. Total cellular protein was then extracted from cells using the immunoprecipitation assay lysis buffer and protein concentrations were assessed using bicinchoninic acid (BCA) kit (Cwbiotech, Beijing,China).Protein samples $(20 \mu \mathrm{g})$ were heated at $100^{\circ} \mathrm{C}$ for $10 \mathrm{~min}$ and then separated in 10\% Tris-Tricine sodium dodecyl sulfate polyacrylamide gel electrophoresis (SDS-PAGE) gel and transferred onto a polyvinylidenedifluoride (PVDF) membrane (EMD Millipore, Billerica, MA, USA). For western blotting, the membranes were blocked for $2 \mathrm{~h}$ at the room temperature in $5 \%$ bovine serum albumin (BSA) and then incubated with primary antibodies against B-cell lymphoma-2 (Bcl-2) (1:1,000), caspase-3 (1:1,000), cleaved poly-adenosine diphosphate-ribose polymerase (cPARP) $(1: 1,000)$ (Proteintech Group, Inc., Cambridge, MA, USA), phosphorylated and total extracellular signal-regulated kinase (ERK) (1:1,000; Cell Signaling Technology, Inc., Shanghai, China), basic fibroblast growth factor (bFGF) $(1: 1,000)$ or vascular endothelial growth factor (VEGF) $(1: 1,000)$ (both from Affinity Biosciences, Cincinnati, OH, USA) for $12 \mathrm{~h}$ at $4^{\circ} \mathrm{C}$. On the next day, the membranes were washed with Tris-based saline-Tween-20 solution (TBST) for three times and then incubated with a secondary antibody at a dilution of 1:1,000 (EMD Millipore) for $1 \mathrm{~h}$ at room temperature. Protein bands were visualized using enhanced chemiluminescence (ECL)-Plus Western blotting detection reagents (Millipore).

Statistical analysis. All experiments were repeated at least three times and the data were expressed as mean \pm standard deviation. All statistical analyses were performed by using SPSS 19.0 software (IBM Corp., Armonk, NY, USA). Student's t-test was used to analyze the mean difference between two groups, while comparisons of the means among multiple groups were analyzed using one-way analysis of variance followed by the post-hoc Student-Newman-Keuls test. The $\mathrm{IC}_{50}$ and combination index values were calculated using Compusyn software version 1.0 (ComboSyn, Inc.). A P-value equal to or $<0.05$ was considered statistically significant. 

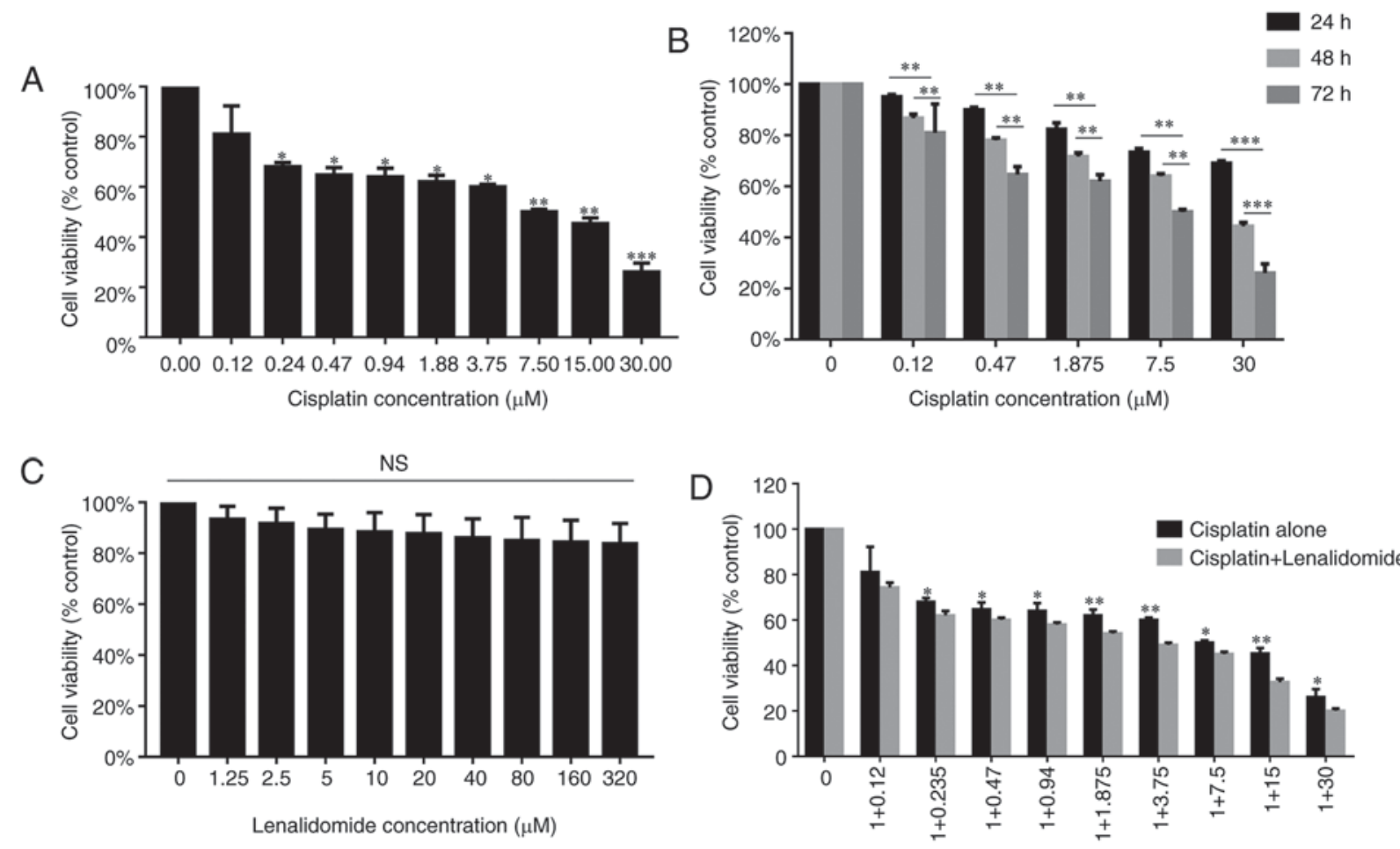

Cisplatin vs lenalidomide at $1+$ cisplatin concentration $(\mu \mathrm{M})$

Figure 1. Effect of lenalidomide and cisplatin and their combination on MDA-MB-231 cell viability in vitro. MDA-MB-231 cells were grown and treated with (A) cisplatin at varying concentrations for $72 \mathrm{~h} .{ }^{*} \mathrm{P}<0.05,{ }^{* * *} \mathrm{P}<0.01$ and ${ }^{* * *} \mathrm{P}<0.001$ vs. $0.0 \mu \mathrm{M}$ cisplatin group; (B) cisplatin at 24,48 and $72 \mathrm{~h}$; (C) lenalidomide; or (D) their combination up to $72 \mathrm{~h}$ and then subjected to cell viability MTT assay. $\mathrm{P}<0.05,{ }^{* * *} \mathrm{P}<0.01$ and ${ }^{* * * *} \mathrm{P}<0.001$ vs. corresponding cisplatin + lenalidomide group. NS, no significant difference.

\section{Results}

Lenalidomide enhanced the cytotoxic effect of cisplatin on $M D A-M B-231$ cells. We first assessed the effects of lenalidomide and cisplatin combination on MDA-MB-231 cell viability using the MTT sassy. Our results showed that cisplatin had a dramatic effect on the viability of MDA-MB-231 cells with an $\mathrm{IC}_{50}$ of $7.8 \mu \mathrm{M}$ (Fig. $1 \mathrm{~A}$ ); in addition, MDA-MB-231 cells viability was decreased with increasing the incubation time from 24 to 72 h. At 72 h, MDA-MB-231 cell viability was decreased to the minimum (Fig. 1B). However, lenalidomide treatment alone had a minimal effect on MDA-MB-231 cell viability, up to $320 \mu \mathrm{M}$ (Fig. 1C). The maximum concentration of lenalidomide that could be safely used in patients is $1 \mu \mathrm{M}$; thus, we combined lenalidomide at $1 \mu \mathrm{M}$ with nine concentrations of cisplatin in MDA-MB-231 cells. As a result, cell viability was dramatically reduced in combined group as compared to cisplatin alone (Fig. 1D). The effect of drug combination was assessed by Compusyn software. $\mathrm{IC}_{50}$ is the major evaluation index in Compusyn software. The $\mathrm{IC}_{50}$ of cisplatin was reduced to $3.0 \mu \mathrm{M}$ in combined treatment group. Therefore, this concentration was further applied in apoptosis experiments.

Combination of lenalidomide and cisplatin increased cell apoptosis in MDA-MB-231 cells. We then treated MDA-MB-231 cells with $1 \mu \mathrm{M}$ lenalidomide, $3.0 \mu \mathrm{M}$ cisplatin, or combination and cell apoptosis was measured using FITC Annexin V-FITC/PI apoptosis detection kit. Our results demonstrated that lenalidomide and cisplatin alone induced cell apoptosis $(\mathrm{P}<0.05)$. In addition, their combination significantly increased cell apoptosis by 1.60 and 1.38 -folds compared to lenalidomide and cisplatin single drug treatment $(\mathrm{P}<0.01)$ (Fig. 2). The combination treatment increased the rate of early apoptosis by 1.41 and 1.27 -folds as well as the rate of later apoptosis by 1.80 and 1.51 -folds compared to lenalidomide and cisplatin treatments, respectively. This suggested that lenalidomide and cisplatin combination reduces MDA-MB-231 cell viability through the induction of cell apoptosis.

Changes in protein expression after lenalidomide, cisplatin, or their combination treatment of TNBC cells in vitro. We further assessed the modulation in protein expression in MDA-MB-231 cells upon treating with 1 lenalidomide and cisplatin combination. We found that lenalidomide treatment alone was able to reduce the level of phosphorylated (p)-ERK, which is known to regulate cell proliferation, in MDA-MB-231 cells, whereas p-ERK level was induced by cisplatin treatment alone (Fig. 3). Their combination significantly reduced the p-ERK level in MDA-MB-231 cells compared to cisplatin alone. Furthermore, lenalidomide and cisplatin alone downregulated the expression of anti-apoptotic protein Bcl-2 but upregulated expression of pro-apoptotic protein caspase-3 and PARP in MDA-MB-231 cells (Fig. 3). However, the combination further inhibited $\mathrm{Bcl}-2$ expression and upregulated caspase-3 and cleaved PARP expression compared to single treatment (Fig. 3).

Lenalidomide plays a key role in tumor progression through the inhibition of angiogenesis. VEGF and bFGF are the most potent mediators in angiogenesis in human cells. We, 

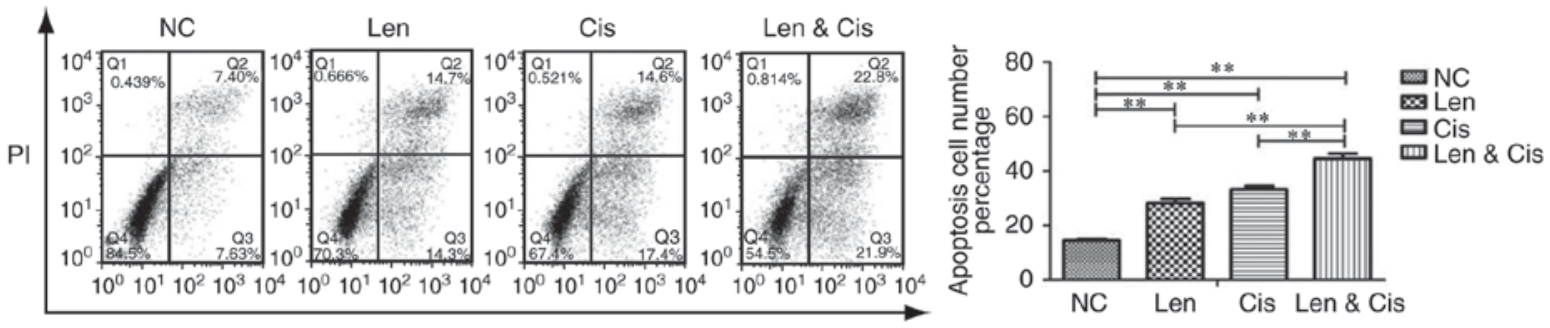

AnnexinV FITC

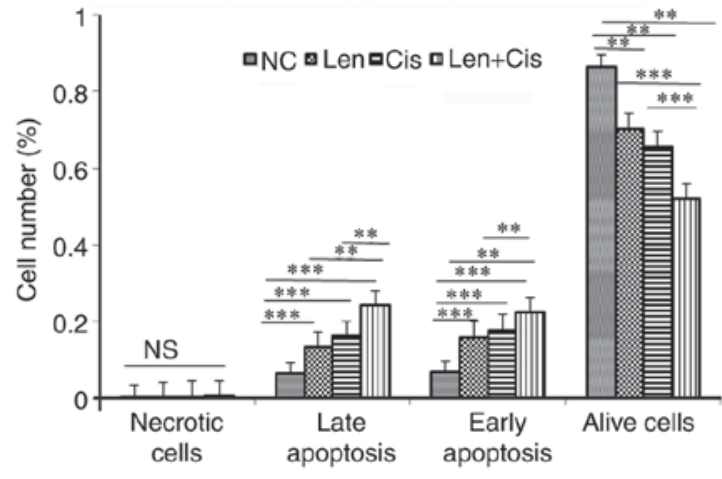

Affect of lenalidomide and cisplatin on apoptosis

Figure 2. Effect of lenalidomide and cisplatin on MDA-MB-231 cell apoptosis in vitro. MDA-MB-231 cells were grown and treated with lenalidomide, cisplatin, or their combination for $72 \mathrm{~h}$ and apoptotic cells were detected using FITC Annexin V-FITC/PI apoptosis detection kit. The bar graph is a quantitative presentation of the flow cytometric data. ${ }^{* *} \mathrm{P}<0.01$ and ${ }^{* * *} \mathrm{P}<0.001$. NS, no significant difference; NC, negative control; Len, lenalidomide; Cis, cisplatin; FITC, fluorescein isothiocyanate; PI, propidium iodide; NC, negative control.

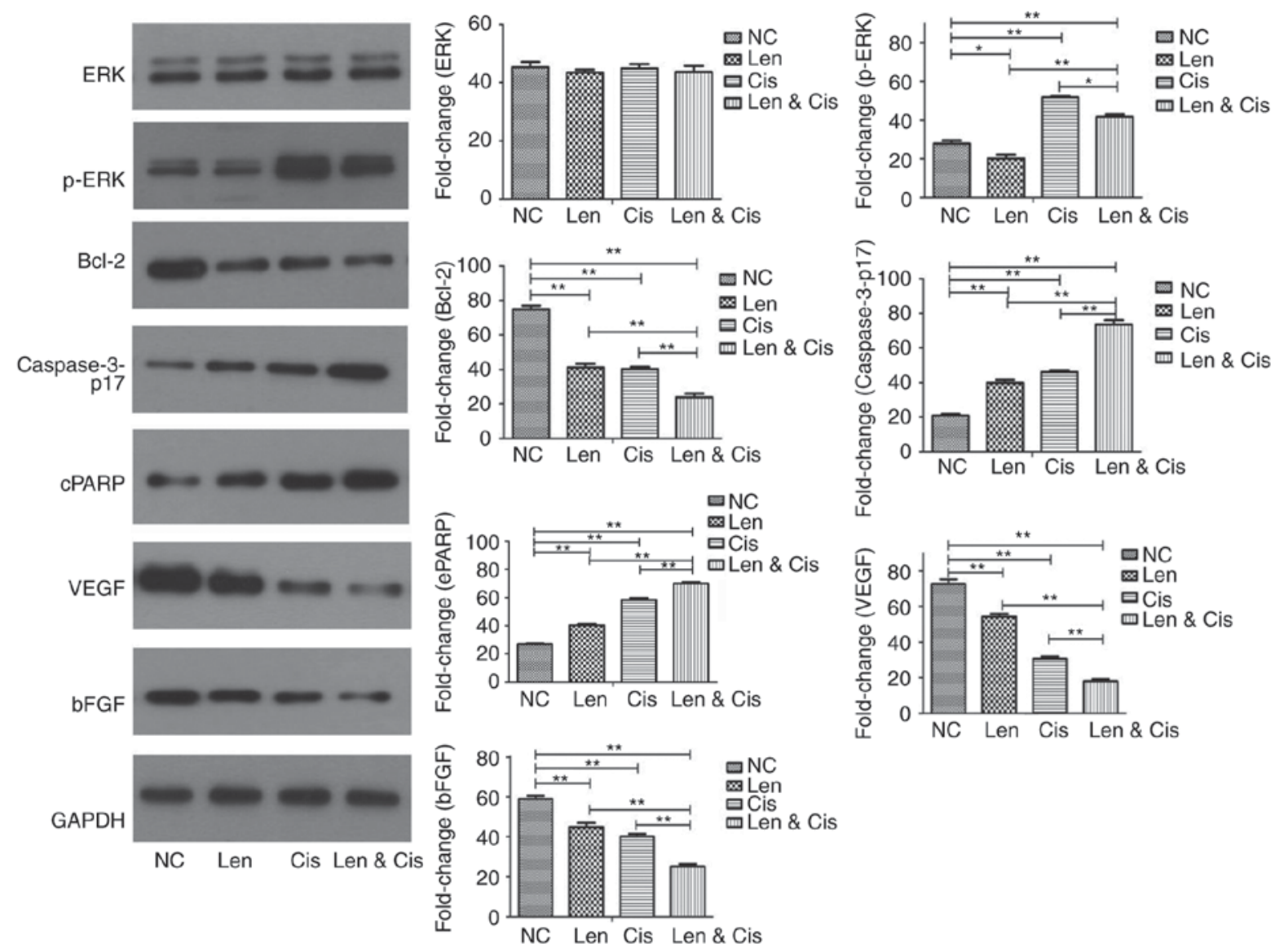

Figure 3. Effect of lenalidomide and cisplatin on modulating the different protein expression in MDA-MB-231 cells. MDA-MB-231 cells were grown and treated with lenalidomide, cisplatin, or their combination for $72 \mathrm{~h}$ and then subjected to western blot analysis. The bar graphs are a quantitative presentation of the western blot analyses. ${ }^{*} \mathrm{P}<0.05$ and ${ }^{* *} \mathrm{P}<0.01$. ERK, extracellular signal-regulated kinase; p-, phosphorylated; Bcl-2, B-cell lymphoma-2; cPARP, cleaved poly-adenosine diphosphate-ribose polymerase; VEGF, vascular endothelial growth factor; bFGF, basic fibroblast growth factor; NC, negative control. 
therefore, assessed their levels in MDA-MB-231 cells after treating with lenalidomide, cisplatin or combination. Our data showed that lenalidomide or cisplatin alone was able to reduce the levels of VEGF and bFGF proteins in MDA-MB-231 cells, which were further reduced with the combination (Fig. 3).

\section{Discussion}

To date, surgery, chemotherapy, and radiotherapy are the main therapeutic strategies in TNBC. However, a considerable number of TNBC patients are diagnosed at advanced stages of the disease, leading to curable surgery inaccessible because TNBC occurs more often in young women and is prone to early metastasis. Thus, present selections for TNBC treatment are limited and novel therapeutic strategies could help medical oncologists to cure TNBC or improve and prolong survival rates in TNBC patients. The present study assessed the in vitro antitumor activity of lenalidomide and cisplatin combination in TNBC cells. Our data showed that cisplatin alone showed inhibitory effect on MDA-MB-231 cell viability, whereas lenalidomide alone had only minimal effect, even at very high concentrations that are far beyond the clinically achievable dose in humans (16). Moreover, cell apoptosis was significantly induced in MDA-MB-231 cells upon treating with lenalidomide and cisplatin combination. Due to the potential significance of lenalidomide and cisplatin combination in MDA-MB-231, it was also necessary to explore the mechanism of their action in TNBC cells. We found that the combination dramatically reduced the levels of p-ERK, VEGF, bFGF and Bcl-2 proteins $(\mathrm{P}<0.05)$, and upregulated caspase- 3 activity and cleaved PARP expression. This study, therefore, provides preliminary data to support lenalidomide combination with cisplatin in TNBC treatment.

As a single agent, cisplatin is one of the common chemotherapeutic drugs used in various human cancers, especially TNBC (18) and has been shown to inhibit tumor cell proliferation. In the present study, cisplatin significantly reduced MDA-MB-231 cell viability at an $\mathrm{IC}_{50}$ value of $7.8 \mu \mathrm{M}$. On the other hand, it is controversy and debatable whether lenalidomide can be used to control solid tumors $(14,16)$, although lenalidomide significantly inhibited growth of the chronic lymphocytic leukemia cells (19). In the present study, we further confirmed that lenalidomide alone had only a minimal effect on MDA-MB-231 cell viability, even at a very high dose of $320 \mu \mathrm{M}$, far beyond the clinically achievable concentration in humans (20). Thus, it is suggested that lenalidomide alone does not have much anti-proliferative activity in solid tumors, especially in TNBC cells. However, the combination of cisplatin with lenalidomide (at $1 \mu \mathrm{M}$ ) showed a synergetic inhibitory effect on MDA-MB-231 cells as compared to cisplatin alone. Furthermore, cisplatin $\mathrm{IC}_{50}$ value was reduced from $7.8 \mu \mathrm{M}$ to $3 \mu \mathrm{M}$ when combined with $1 \mu \mathrm{M}$ lenalidomide. To achieve the same inhibitory effect on MDA-MB-231 cell, cisplatin dose was significantly reduced when combined with lenalidomide and, thus, cisplatin side effects were also reduced.

Furthermore, we examined the ability of cisplatin, lenalidomide, and their combination in the induction of MDA-MB-231 cell apoptosis in vitro. Czarnomysy et al (21) reported that cisplatin at concentrations 25,50 and $100 \mu \mathrm{M}$ was found to induce apoptosis of MDA-MB-231 cells after $24 \mathrm{~h}$ incubation. In the present study, we investigated whether cisplatin at a lower concentration $3 \mu \mathrm{M}$ could induce MDA-MB-231 cell apoptosis. It is supportive that lenalidomide at the $1 \mu \mathrm{M}$ dose was also able to induce apoptosis of MAD-MB-231 cells or other cancer cell lines $(16,22)$. However, to date, there was no study reporting the effect of combined lenalidomide with cisplatin on TNBC cells. In the present study, we investigated the significant increase in apoptosis after combined treatment, compared with cisplatin and lenalidomide alone. However, further investigation using different TNBC cell lines is required to confirm our current data.

In addition, the present study also illustrated the underlying molecular events on lenalidomide and cisplatin modulation of different cell growth, apoptosis and angiogenesis-related proteins. For example, the ERK1/2 pathway is the classic cell growth signaling of the mitogen-activated protein kinase (MAPK) pathway. Activated ERK1/2 will promote cell survival and proliferation by phosphorylating many nuclear transcription factors (23). The present study showed that lenalidomide alone or in combination with cisplatin could reduce p-ERK levels in TNBC cells, although cisplatin alone induced p-ERK level. Indeed, Fryer et al (24) also reported that lenalidomide significantly reduced level of $\mathrm{p}$-ERK protein in pancreatic cancer cell lines, while Chen et al (25) demonstrated that cisplatin had no effect on $\mathrm{p}$-ERK protein expression in hepatocellular carcinoma cells. Furthermore, it is well established that a decrease in cell apoptosis could contribute to human carcinogenesis (26). The B-cell/leukemia-2 (Bcl-2), cleaved PARP and caspase-3 proteins were reported as the key proteins in regulating cell apoptosis. Decreased Bcl-2 or increased cleaved PARP and caspase-3 will promote cell apoptosis (27). In the present study, we found that lenalidomide and cisplatin induces caspases-3 activity and cleaved PARP expression, while reduces Bcl-2 expression. Our findings are consistent with a previous study (28). However, this study does have some limitations; for example, we only analyzed the expression of caspase- 3 and cleaved poly-adenosine diphosphate-ribose polymerase (cPARP) and whether this combination may affect other apoptosis-related genes is not clear and needs further analysis. In addition, inhibition of tumor angiogenesis was one of the main mechanisms by which lenalidomide mediate its effect in multiple myeloma (7). Lenalidomide is a potent inhibitor of VEGF and bFGF (29), which is confirmed by the present study. VEGF and bFGF are the core factors in promoting tumor angiogenesis.

In conclusion, the combination of lenalidomide and cisplatin demonstrated a synergetic antitumor effect on MDA-MB-231 cells in vitro. This effect was at least partially due to induction of tumor cell apoptosis. Moreover, the inhibition of VEGF and bFGF by lenalidomide could partially contribute to the synergetic effect of lenalidomide and cisplatin combination, suggesting the potential strategy of lenalidomide and cisplatin combination in TNBC treatment.

\section{Acknowledgements}

Not applicable.

\section{Funding}

No funding was received. 


\section{Availability of data and materials}

The datasets used and/or analyzed during the present study are available from the corresponding author on reasonable request.

\section{Authors' contributions}

JL and XWW conceived and designed the experiments. LLY, XMW and QHL performed the experiments. LLY and XMW analyzed the data. LLY and XWW wrote the manuscript.

\section{Ethics approval and consent to participate}

Not applicable.

\section{Consent for publication}

Not applicable.

\section{Competing interests}

The authors confirm that they have no competing interests.

\section{References}

1. Goldhirsch A, Winer EP, Coates AS, Gelber RD Piccart-Gebhart M, Thürlimann B and Senn HJ; Panel members: Personalizing the treatment of women with early breast cancer: Highlights of the St Gallen international expert consensus on the primary therapy of early breast cancer 2013. Ann Oncol 24: 2206-2223, 2013.

2. Carlson RW, Allred DC, Anderson BO, Burstein HJ, Carter WB, Edge SB, Erban JK, Farrar WB, Goldstein LJ, Gradishar WJ, et al: Breast cancer. Clinical practice guidelines in oncology. J Natl Compr Canc Netw 7: 122-192, 2009.

3. Romond EH, Perez EA, Bryant J, Suman VJ, Geyer CE Jr, Davidson NE, Tan-Chiu E, Martino S, Paik S, Kaufman PA, et al: Trastuzumab plus adjuvant chemotherapy for operable HER2-positive breast cancer. N Engl J Med 353: 1673-1684, 2005.

4. Burstein HJ, Temin S, Anderson H, Buchholz TA, Davidson NE, Gelmon KE, Giordano SH, Hudis CA, Rowden D, Solky AJ, et al: Adjuvant endocrine therapy for women with hormone receptor-positive breast cancer: American society of clinical oncology clinical practice guideline focused update. J Clin Oncol 32: 2255-2269, 2014.

5. Yuan N, Meng M, Liu C, Feng L, Hou L, Ning Q, Xin G, Pei L, Gu S, Li X and Zhao X: Clinical characteristics and prognostic analysis of triple-negative breast cancer patients. Mol Clin Oncol 2: 245-251, 2014.

6. Anders $\mathrm{C}$ and Carey LA: Understanding and treating triple-negative breast cancer. Oncology (Williston Park) 22: 1233-1240, 1243, 2008.

7. McCarthy PL, Owzar K, Hofmeister CC, Hurd DD, Hassoun H, Richardson PG, Giralt S, Stadtmauer EA, Weisdorf DJ, Vij R, et al: Lenalidomide after stem-cell transplantation for multiple myeloma. N Engl J Med 366: 1770-1781, 2012.

8. Teo SK: Properties of thalidomide and its analogues: Implications for anticancer therapy. AAPS J 7: E14-E19, 2005.

9. Wu L, Parton A, Lu L, Adams M, Schafer P and Bartlett JB: Lenalidomide enhances antibody-dependent cellular cytotoxicity of solid tumor cells in vitro: Influence of host immune and tumor markers. Cancer Immunol Immunother 60: 61-73, 2011.

10. Qu Z, Jiang C, Wu J and Ding Y: Lenalidomide induces apoptosis and inhibits angiogenesis via caspase-3 and VEGF in hepatocellular carcinoma cells. Mol Med Rep 14: 4781-4786, 2016.

11. Weber DM, Chen C, Niesvizky R, Wang M, Belch A, Stadtmauer EA, Siegel D, Borrello I, Rajkumar SV, Chanan-Khan AA, et al: Lenalidomide plus dexamethasone for relapsed multiple myeloma in North America. N Engl J Med 357: 2133-2142, 2007
12. Wu L, Liu W, Galustian C, Schafer P, Dalgleish AG and Bartlett JB: Effect of lenalidomide on the antiproliferative effect of gemcitabine against pancreatic tumor cells and on immune-mediated pancreatic cancer cell death. J Clin Oncol 27 (Suppl 15): e14635, 2009.

13. Said R, Ye Y, Hong DS, Naing A, Falchook G, Fu S, Wheler JJ, Piha-Paul S and Tsimberidou AM: Phase I clinical trial of lenalidomide in combination with 5-fluorouracil, leucovorin, and oxaliplatin in patients with advanced cancer. Cancer Chemother Pharmacol 77: 575-581, 2016.

14. Safran H, Charpentier KP, Kaubisch A, Mantripragada K, Dubel G, Perez K, Faricy-Anderson K, Miner T, Eng Y, Victor J, et al: Lenalidomide for second-line treatment of advanced hepatocellular cancer: A Brown University oncology group phase II study. Am J Clin Oncol 38: 1-4, 2015.

15. Henry JY, Lu L, Adams M, Meyer B, Bartlett JB, Dalgleish AG and Galustian C: Lenalidomide enhances the anti-prostate cancer activity of docetaxelin vitro and in vivo.Prostate 72: 856-867, 2012.

16. Brosseau C, Colston K, Dalgleish AG and Galustian C: The immunomodulatory drug lenalidomide restores a vitamin D sensitive phenotype to the vitamin $\mathrm{D}$ resistant breast cancer cell line MDA-MB-231 through inhibition of BCL-2: Potential for breast cancer therapeutics. Apoptosis 17: 164-173, 2012.

17. Chou TC and Talalay P: Quantitative analysis of dose-effect relationships: The combined effects of multiple drugs or enzyme inhibitors. Adv Enzyme Regul 22: 27-55, 1984.

18. Jovanović B, Mayer IA, Mayer EL, Abramson VG, Bardia A, Sanders M, Kuba MG, Estrada MV, Beeler JS, Shaver TM, et al: A randomized phase II neoadjuvant study of cisplatin, paclitaxel with or without everolimus in patients with stage II/III triple-negative breast cancer (TNBC): Responses and long-term outcome correlated with increased frequency of DNA damage response gene mutations, TNBC subtype, AR status, and Ki67. Clin Cancer Res 23: 4035-4045, 2017.

19. Fecteau JF, CorralLG, Ghia EM, Gaidarova S, Futalan D, Bharati IS, Cathers B, Schwaederlé M, Cui B, Lopez-Girona A, et al: Lenalidomide inhibits the proliferation of CLL cells via a cereblon/p21(WAF1/Cip1)-dependent mechanism independent of functional p53. Blood 124: 1637-1644, 2014.

20. Bridoux F, Chen N, Moreau S, Arnulf B, Moumas E, Abraham J, Desport E, Jaccard A and Fermand JP: Pharmacokinetics, safety, and efficacy of lenalidomide plus dexamethasone in patients with multiple myeloma and renal impairment. Cancer Chemother Pharmacol 78: 173-182, 2016.

21. Czarnomysy R, Surażyński A, Popławska B, Rysiak E, Pawłowska N, Czajkowska A, Bielawski K and Bielawska A: Synergistic action of cisplatin and echistatin in MDA-MB-231 breast cancer cells. Mol Cell Biochem 427: 13-22, 2017.

22. Jin Z, Qing K, Ouyang Y, Liu Z, Wang W, Li X, Xu Z and Li J: Low dose of lenalidmide and PI3K/mTOR inhibitor trigger synergistic cytoxicity in activated B cell-like subtype of diffuse large B cell lymphoma. J Exp Clin Cancer Res 35: 52, 2016.

23. Liu Y, Bi T, Wang G, Dai W, Wu G, Qian L, Gao Q and Shen G: Lupeol inhibits proliferation and induces apoptosis of human pancreatic cancer PCNA-1 cells through AKT/ERK pathways. Naunyn Schmiedebergs Arch Pharmacol 388: 295-304, 2015.

24. Fryer RA, Barlett B, Galustian C and Dalgleish AG: Mechanisms underlying gemcitabine resistance in pancreatic cancer and sensitisation by the $\mathrm{iMiD}^{\mathrm{TM}}$ lenalidomide. Anticancer Res 31: 3747-3756, 2011.

25. Chen FS, Cui YZ, Luo RC, Wu J and Zhang H: Coadministration of sorafenib and cisplatin inhibits proliferation of hepatocellular carcinoma HepG2 cells in vitro. Nan Fang Yi Ke Da Xue Xue Bao 28: 1684-1687, 2008 (In Chinese).

26. Bold RJ, Termuhlen PM and McConkey DJ: Apoptosis, cancer and cancer therapy. Surg Oncol 6: 133-142, 1997.

27. Wu J, Cai Y, Li M, Zhang Y, Li H and Tan Z: Oxymatrine promotes S-phase arrest and inhibits cell proliferation of human breast cancer cells in vitro through mitochondria-mediated apoptosis. Biol Pharm Bull 40: 1232-1239, 2017.

28. Munugalavadla V, Mariathasan S, Slaga D, Du C, Berry L, Del Rosario G, Yan Y, Boe M, Sun L, Friedman LS, et al: The PI3K inhibitor GDC-0941 combines with existing clinical regimens for superior activity in multiple myeloma. Oncogene 33: 316-325, 2014.

29. Yang XW, Ma LM, Zhao XQ and Ruan LH: Clinical curative efficacy of lenalidomide combined with chemotherapy for acute leukemia and its impact on VEGF. Zhongguo Shi Yan Xue Ye Xue Za Zhi 24: 702-706, 2016 (In Chinese). 